\title{
Thermodynamic Instability of 3D Einstein-Born-Infeld AdS Black Holes
}

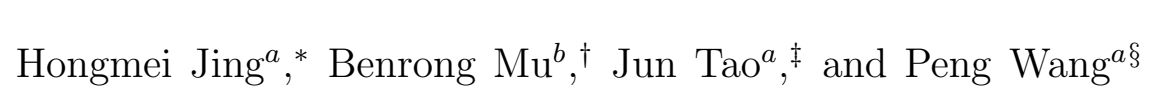 \\ ${ }^{a}$ Center for Theoretical Physics, College of Physics, \\ Sichuan University, Chengdu, 610065, China and \\ ${ }^{b}$ Physics Teaching and Research Section, College of Medical Technology, \\ Chengdu University of Traditional Chinese Medicine, Chengdu 611137, China
}

\begin{abstract}
Super-entropic black holes possess finite-area but noncompact event horizons and violate the reverse isoperimetric inequality. It has been conjectured that such black holes always have negative specific heat at constant volume $C_{V}$ or negative specific heat at constant pressure $C_{P}$ whenever $C_{V}>0$, making them unstable in extended thermodynamics. In this paper, we test this instability conjecture on a family of nonlinear electrodynamics black holes, namely 3D Einstein-Born-Infeld (EBI) AdS black holes. Our results show that when nonlinear electrodynamics effects are weak, the instability conjecture is valid. However, the conjecture can be violated in some parameter region when nonlinear electrodynamics effects are strong enough. This observation thus provides a counter example to the instability conjecture, which suggests that super-entropic black holes could be thermodynamically stable.
\end{abstract}

\footnotetext{
*Electronic address: hmjing@stu.scu.edu.cn

†Electronic address: benrongmu@cdutcm.edu.cn

${ }^{\ddagger}$ Electronic address: taojun@scu.edu.cn, Corresponding author

$\S$ Electronic address: pengw@scu.edu.cn
} 


\section{INTRODUCTION}

Black hole thermodynamics has been developed on the understanding of black holes and quantum physics [1 1 5]. In the extended phase space, the cosmological constant can be considered as a thermodynamic variable [6-8]. Specifically, one can treat the cosmological constant $\Lambda$ as a thermodynamic pressure $P=-\frac{\Lambda}{8 \pi G}$, which gives that its conjugate quantity is interpreted as a thermodynamic volume. For a negative cosmological constant $\Lambda$, the pressure $P$ is positive, which yields a well-defined equilibrium thermodynamic framework. It is worth noting that this volume is usually not equal to the geometric volume of black holes except in some simple cases, such as AdS Schwarzschild black holes.

Although the definition of thermodynamic volume has been given, its physical interpretation is still a puzzle. An early attempt to answer this question leads to the conjecture that the volume satisfies the reverse isoperimetric inequality [9, 10]. The conjecture was motivated in the progress of studying Kerr-AdS black holes and then generalized to other black holes. The reverse isoperimetric inequality is saturated for Schwarzschild-AdS black holes, which indicates that for a black hole of a given thermodynamic volume $V$, the entropy is maximized for Schwarzschild-AdS black holes [9]. However, further investigations discovered that this inequality does not apply to all kinds of black holes. Those black holes that exceed the maximum entropy bound are called super-entropic black holes [11 15], which are rotating with non-compact event horizons of finite surface area [11]. More relevant discussions can be found in refs. [16 29].

A black hole reduces its mass-energy via the Hawking radiation. If the specific heat is negative, this shrinking would lead to a higher temperature, increased radiation, and hence more mass loss. Therefore, the system accelerates through this downward spiral instead of settling into an equilibrium state. For charged BTZ black holes, which are the simplest super-entropic black holes, it has been shown that there is a connection between the violation of the reverse isoperimetric inequality and the thermodynamical instability with the specific heat at constant volume $C_{V}<0[23]$. This result consequently leads to a natural conjecture that super-entropic black holes always have $C_{V}<0$, making them unstable in the extended thermodynamics. Later, it was found that this conjecture is violated for generalized exotic BTZ black holes in some parameter region [24]. However in this case, the specific heat at constant pressure $C_{P}$ was exhibited to be negative whenever $C_{V}>0$. Thus, a broader 
version of the instability conjecture was proposed [24], which states that all super-entropic black holes are in general thermodynamically unstable with either negative $C_{V}$ or negative $C_{P}$. The instability conjecture was analytically verified for $3 \mathrm{D}$ charged BTZ black holes [23, 30, 31]. Since it is difficult to obtain analytical expressions for $C_{V}$ and $C_{P}$, using methods of ref. [32], the instability conjecture was numerically tested for ultra spinning $d$ dimensional Kerr black holes [14], generalized exotic BTZ black holes [24] and super-entropic black hole with Immirzi in ref. [27].

Here, we test the instability conjecture on EBI AdS black holes in $(2+1)$-dimensional space-time. An EBI AdS black hole is the charged black hole solution in the EBI theory based on the non-linear electrodynamics proposed by Born and Infeld in 1934 [33], and is an extension of a RN black hole in the Einstein-Maxwell theory. Since it was found that the non-linear electrodynamics, in particular the Born-Infeld electrodynamics, can come from the low-energy limit of string theory and encodes the low-energy dynamics of D-branes (i.e., the low-energy effective action for a constant electromagnetic field is precisely the Born-Infeld action) [34 37], it has attracted considerable attention in recent years. After BI black hole solutions in anti-de Sitter space were obtained [38, 39], their properties have been extensively investigated [40-57]. Although various aspects of 3D EBI AdS black holes have also been studied, the specific heat and instability conjecture are expected to explore.

The organization of the rest of this work is as follows. In section II, we discuss thermodynamic quantities of 3D EBI AdS black holes. In section III, we first show that 3D EBI AdS black holes violate the reverse isoperimetric inequality, and hence are super-entropic. Then, the instability conjecture is considered by calculating $C_{V}$ and $C_{P}$. We find that when nonlinear electrodynamics effects are strong enough, there exists some parameter region where $C_{V}$ and $C_{P}$ are both positive. This observation provides a counter example to the instability conjecture. The conclusion is given in section IV. In this paper, we use geometrical units where $G, c, \hbar$, and $k_{B}$ have been set to unity. 


\section{THERMODYNAMICS OF 3D EBI ADS BLACK HOLES}

In this section, thermodynamics of 3D EBI AdS black holes is discussed. The action of the 3D-Einstein gravity coupled with the Born-Infeld electrodynamics is

$$
\begin{aligned}
I & =\int d^{3} x \sqrt{-g}\left[\frac{R-2 \Lambda}{16 \pi}+L(F)\right], \\
L(F) & =\frac{b^{2}}{4 \pi}\left(1-\sqrt{1+\frac{2 F}{b^{2}}}\right) .
\end{aligned}
$$

Here, the constant $b$ is the Born-Infeld parameter, $g$ is the determinant of the metric tensor, $\Lambda=-1 / l^{2}$ is the cosmological constant, $l$ is the AdS radius, and $L(F)$ is the Lagrangian of the Born-Infeld electrodynamics. The metric and gauge potential are [40, 41]

$$
\begin{aligned}
d s^{2} & =-f(r) d t^{2}+f(r)^{-1} d r^{2}+r^{2} d \theta^{2}, \\
f(r) & =-8 M+\frac{r^{2}}{l^{2}}+2 b^{2} r\left(r-\sqrt{r^{2}+\frac{Q^{2}}{4 b^{2}}}\right)-\frac{1}{2} Q^{2} \ln \left[r+\sqrt{r^{2}+\frac{Q^{2}}{4 b^{2}}}\right] \\
& +\frac{1}{2} Q^{2} \ln \left[l+\sqrt{l^{2}+\frac{Q^{2}}{4 b^{2}}}\right]-2 b^{2} l\left[l-\sqrt{l^{2}+\frac{Q^{2}}{4 b^{2}}}\right],
\end{aligned}
$$

where $Q$ and $M$ stand for the charge and mass of EBI black holes, respectively. In the limit of $b \rightarrow \infty$, it reduces to the charged BTZ black hole solution [23],

$$
f^{\mathrm{BTZ}}(r)=-8 M-\frac{Q^{2}}{2} \log \left(\frac{r}{l}\right)+\frac{r^{2}}{l^{2}} .
$$

The horizon is located at $r=r_{+}$with $f\left(r_{+}\right)=0$, from which the mass of 3D EBI AdS black holes is obtained [40],

$$
\begin{aligned}
M & =\frac{r_{+}^{2}}{8 l^{2}}+\frac{1}{4} b^{2} r_{+}\left(r_{+}-\sqrt{r_{+}^{2}+\frac{Q^{2}}{4 b^{2}}}\right)-\frac{1}{16} Q^{2} \ln \left[r_{+}+\sqrt{r_{+}^{2}+\frac{Q^{2}}{4 b^{2}}}\right] \\
& +\frac{1}{16} Q^{2} \ln \left[l+\sqrt{l^{2}+\frac{Q^{2}}{4 b^{2}}}\right]-\frac{1}{4} b^{2} l\left[l-\sqrt{l^{2}+\frac{Q^{2}}{4 b^{2}}}\right] .
\end{aligned}
$$

In the extended thermodynamics, one identifies the enthalpy $H$ [6] with the mass of the black hole, and the pressure is $P=-\Lambda / 8 \pi=1 / 8 \pi l^{2}$. Moreover, the entropy $S$ is

$$
S=\frac{A}{4}=\frac{1}{2} \pi r_{+}
$$

The first law of thermodynamics, $d M=T d S+V d P+\Phi d Q$, gives the temperature and the thermodynamic volume of 3D EBI AdS black holes 


$$
\begin{aligned}
& T=\left.\frac{\partial M}{\partial S}\right|_{P}=\frac{r_{+}}{2 \pi l^{2}}+\frac{b^{2} r_{+}}{\pi}\left(1-\sqrt{1+\frac{Q^{2}}{4 b^{2} r_{+}^{2}}}\right), \\
& V=\left.\frac{\partial M}{\partial P}\right|_{S}=\pi r_{+}^{2}+2 \pi l^{4} b^{2}\left(1-\sqrt{1+\frac{Q^{2}}{4 b^{2} l^{2}}}\right),
\end{aligned}
$$

respectively. It is observed that the thermodynamic volume is different from the geometric volume $\pi r_{+}^{2}$.

\section{INSTABILITY CONJECTURE OF 3D EBI ADS BLACK HOLES}

For an asymptotically AdS black hole in the extended phase space, it was conjectured in ref. [9] that a reverse isoperimetric inequality holds,

$$
R \equiv\left(\frac{(d-1) V}{\omega_{d-2}}\right)^{\frac{1}{d-1}}\left(\frac{\omega_{d-2}}{A}\right)^{\frac{1}{d-2}} \geq 1,
$$

where the isoperimetric ratio $R$ is defined. Here, $V$ is the thermodynamic volume, $A$ is the horizon area, $\omega_{d}$ stands for a $d$-dimensional unit sphere,

$$
\omega_{d}=\frac{2 \pi^{\frac{d+1}{2}}}{\Gamma\left(\frac{d+1}{2}\right)},
$$

where $\omega_{1}=2 \pi$ and $\omega_{2}=4 \pi$. The reverse isoperimetric inequality is saturated for a Schwarzschild AdS black hole since its thermodynamic volume simply equals to its naive geometric volume. For some more complicated black holes, e.g., Kerr [9], STU [58] and Taub-NUT/Bolt black holes [59], thermodynamic volumes are larger than naive geometric volumes, hence resulting in $R>1$. Moreover unlike a Schwarzschild AdS black hole, these black holes have nonzero $C_{V}$. However, several black hole solutions were later found to violate the reverse isoperimetric inequality [11 $14,60,61]$. A black hole that violates the inequality is dubbed "super-entropic black hole" since its entropy is larger than the maximum entropy allowed by the reverse isoperimetric inequality. Argued in ref. [11], the violation is attributed to a result of the finite-area but noncompact event horizon. It was further presented in refs. [23, 24] that a large family of super-entropic black holes has $C_{V}<0$ or $C_{P}<0$ whenever $C_{V}>0$, showing that they are unstable in the extended thermodynamics.

In this section, we first show that 3D EBI AdS black holes are super-entropic, which means that they violate the reverse isoperimetric inequality (9). In fact, according to Eqs. (6) , (7) 
and (9), the isoperimetric ratio $R$ for 3D EBI AdS black holes can be readily computed to be

$$
R=\sqrt{1-\frac{l^{2} Q^{2}}{2 r_{+}^{2}}\left(1+\sqrt{1+\frac{Q^{2}}{4 l^{2} b^{2}}}\right)^{-1}}
$$

It is obvious to observe from Eq. (11) that $R<1$, which means 3D EBI AdS black holes violate the reverse isoperimetric inequality as long as $Q \neq 0$. Note that when $Q=0$, EBI AdS black holes would reduce to Schwarzschild AdS black holes, which have $R=$ 1. Consequently, 3D EBI AdS black holes are super-entropic. In the remainder of this section, we discuss behavior of $C_{V}$ and $C_{P}$ of 3D EBI AdS black holes and provide further investigation for the instability conjecture.

Using Eq. (6), we can write thermodynamic quantities in terms of $S$ and $P$,

$$
\begin{aligned}
& T=\frac{8 P S}{\pi}+\frac{2 S b^{2}}{\pi^{2}}\left(1-\sqrt{1+\frac{\pi^{2} Q^{2}}{16 b^{2} S^{2}}}\right), \\
& V=\frac{4 S^{2}}{\pi}+\frac{b^{2}}{32 \pi P^{2}}\left(1-\sqrt{1+\frac{2 \pi P Q^{2}}{b^{2}}}\right) .
\end{aligned}
$$

From Eq. (6), we observe that the entropy $S$ is geometrical, and only depends on the horizon radius $r_{+}$. Hence the entropy $S$ and the thermodynamic volume $V$ are independent functions, which consequently gives a nonzero $C_{V}$. To obtain the specific heat at constant volume $C_{V}$, it will be easier to start with $C_{P}$. Using Eq. (12), we can express $S$ in terms of $T$ and $P$,

$$
S=\frac{\pi T}{16 P}\left[1+\left(1+\frac{2 \pi P}{b^{2}}\right)^{-1}\left(\frac{2 \pi P}{b^{2}}+\sqrt{1+\frac{4 P^{2} Q^{2}}{T^{2} b^{2}}+\frac{2 P Q^{2}}{\pi T^{2}}}\right)\right] .
$$

Then $C_{P}(T)$ is given by

$$
C_{P}(T)=\left.T \frac{\partial S}{\partial T}\right|_{P}=\frac{\pi T}{16 P}\left[1+\left(1+\frac{2 \pi P}{b^{2}}\right)^{-1}\left(\frac{2 \pi P}{b^{2}}+\frac{1}{\sqrt{1+\frac{4 P^{2} Q^{2}}{b^{2} T^{2}}+\frac{2 P Q^{2}}{\pi T^{2}}}}\right)\right],
$$

which is manifestly positive. For large $T$, one has $C_{P}(T)=\frac{\pi T}{8 P}+\cdots$. When $b \rightarrow \infty$, Eq. (15) reduces to $C_{P}^{\mathrm{BTZ}}$ of charged BTZ black holes (see Eq. (7) in ref. [23]),

$$
C_{P}^{\mathrm{BTZ}}(T)=\frac{\pi T}{16 P}\left[1+\frac{1}{\sqrt{1+\frac{2 P Q^{2}}{\pi T^{2}}}}\right] .
$$


One can calculate $C_{V}(T)$ from $C_{P}(T)$ via the well-known relation,

$$
\frac{C_{P}}{C_{V}}=\frac{1}{\kappa_{T} \beta_{S}}
$$

where $\kappa_{T} \equiv-V \partial P /\left.\partial V\right|_{T}$ is the isothermal bulk modulus, and $\beta_{S} \equiv-V^{-1} \partial V /\left.\partial P\right|_{S}$ is the adiabatic compressibility. To be self-contained, a derivation of Eq. (17) is given in the appendix. Substituting Eq. (14) to Eq. (13) yields

$$
\begin{aligned}
V(T, P) & =\frac{\pi T^{2}}{64 P^{2}}\left[1+\left(\frac{2 \pi P}{b^{2}}+1\right)^{-1}\left(\frac{2 \pi P}{b^{2}}+\sqrt{1+\frac{4 P^{2} Q^{2}}{T^{2} b^{2}}+\frac{2 P Q^{2}}{\pi T^{2}}}\right)\right]^{2} \\
& -\frac{Q^{2}}{16 P}\left(\sqrt{1+\frac{2 \pi P Q^{2}}{b^{2}}}+1\right)^{-1} .
\end{aligned}
$$

From Eqs. (13) and (18), $\kappa_{T}$ and $\beta_{S}$ can be readily computed,

$$
\begin{gathered}
\kappa_{T} \equiv-V \partial P /\left.\partial V\right|_{T}=-V\left[\frac{-8 S^{2}}{\pi P}+\frac{T S}{2 P} \gamma+\frac{1}{16 P^{2}} \frac{Q^{2}}{\delta+1}+\frac{\pi Q^{4}}{16 P b^{2}} \frac{1}{\delta(\delta+1)^{2}}\right]^{-1}, \\
\beta_{S} \equiv-V^{-1} \partial V /\left.\partial P\right|_{S}=-V^{-1}\left[\frac{Q^{2}}{8 P^{2}\left(\sqrt{1+\frac{2 \pi P Q^{2}}{b^{2}}}+1\right)}-\frac{Q^{2}}{32 P^{2} \sqrt{1+\frac{2 \pi P Q^{2}}{b^{2}}}}\right],
\end{gathered}
$$

where

$$
\begin{aligned}
& \gamma=\frac{\frac{2 \pi}{b^{2}}+\frac{\eta^{2}-1}{P \eta}-\frac{Q^{2}}{\eta \pi T^{2}}}{\left(\frac{2 \pi P}{b^{2}}+1\right)}-\frac{\left(\frac{2 \pi P}{b^{2}}+\eta\right)\left(\frac{2 \pi}{b^{2}}\right)}{\left(\frac{2 \pi P}{b^{2}}+1\right)^{2}}, \\
& \eta=\sqrt{1+\frac{4 P^{2} Q^{2}}{T^{2} b^{2}}+\frac{2 P Q^{2}}{\pi T^{2}}} \\
& \delta=\sqrt{1+\frac{2 \pi P Q^{2}}{b^{2}}} .
\end{aligned}
$$

With above results for $\kappa_{T}, \beta_{S}$ and $C_{P}(T)$, one can use Eq. (17) to obtain the specific heat at constant volume $C_{V}$. As a check, in the limit of $b \rightarrow \infty$, we find that $C_{V}(T)$ becomes $C_{V}^{\mathrm{BTZ}}(T)$ of charged BTZ black holes (see Eq. (10) in ref. [23]), where

$$
C_{V}^{\mathrm{BTZ}}(T)=-\frac{Q^{2}}{32 T}\left[\frac{1+\sqrt{1+\frac{2 P Q^{2}}{\pi T^{2}}}}{1+\sqrt{1+\frac{2 P Q^{2}}{\pi T^{2}}}+\frac{3 P Q^{2}}{2 \pi T^{2}}}\right] .
$$

In FIG. 1, we plot the specific heat at constant volume $C_{V}$, the specific heat at constant pressure $C_{P}$, the black hole mass $M$ and the black hole temperature $T$ as functions of the 

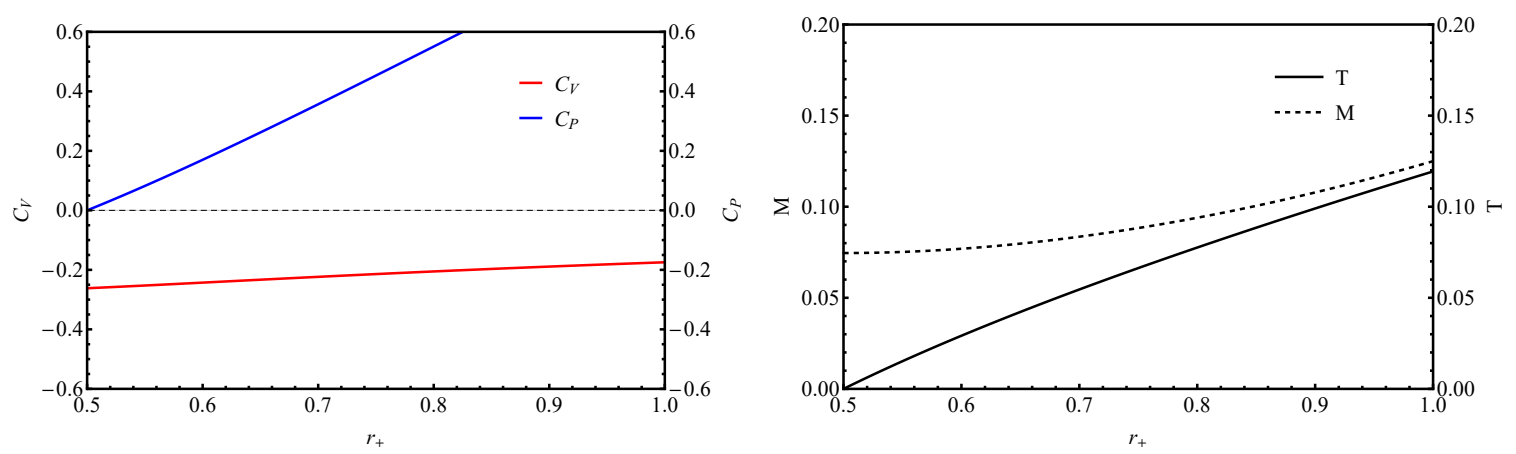

(a) $C_{V} \& C_{P}$ vs. $r_{+}$and $M \& T$ vs. $r_{+}$for $b=100$.
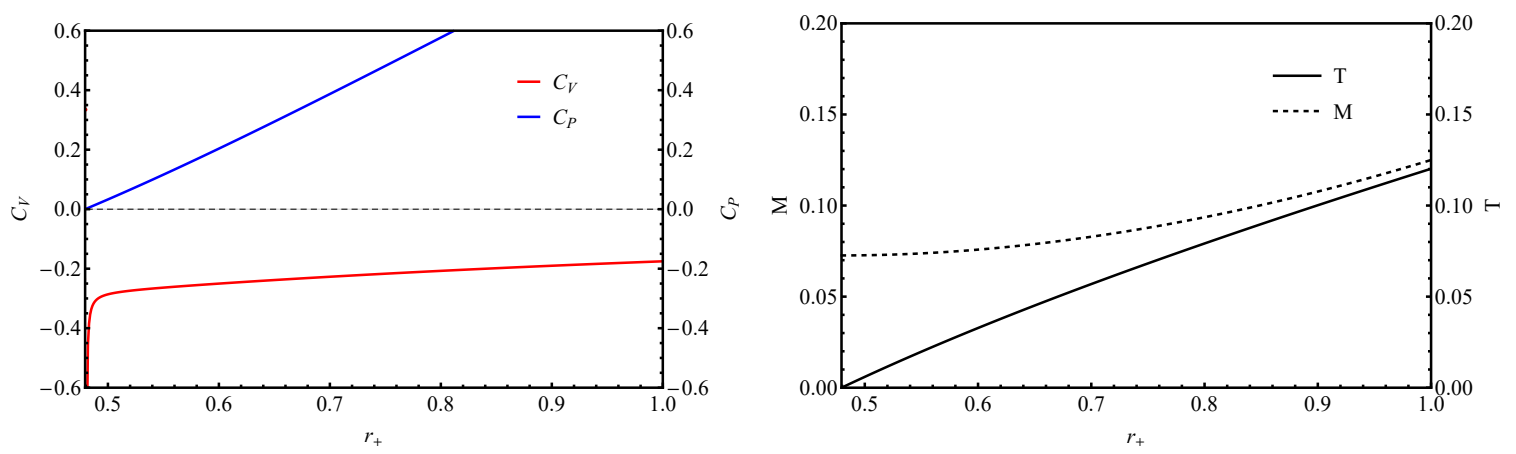

(b) $C_{V} \& C_{P}$ vs. $r_{+}$and $M \& T$ vs. $r_{+}$for $b=1.69$.
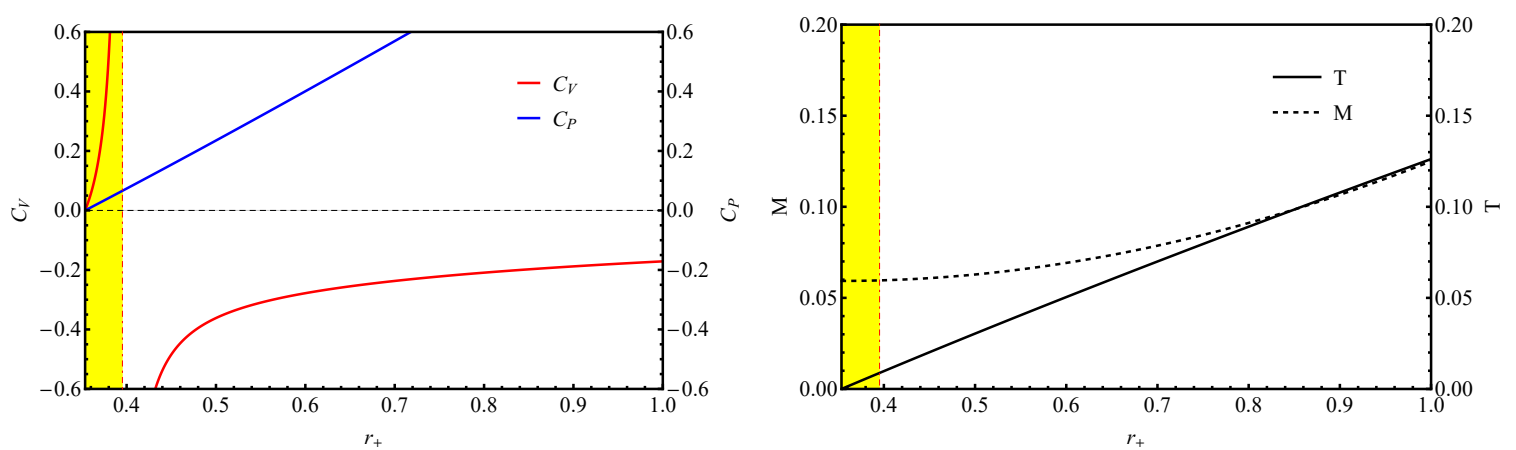

(c) $C_{V} \& C_{P}$ vs. $r_{+}$and $M \& T$ vs. $r_{+}$for $b=0.5$.
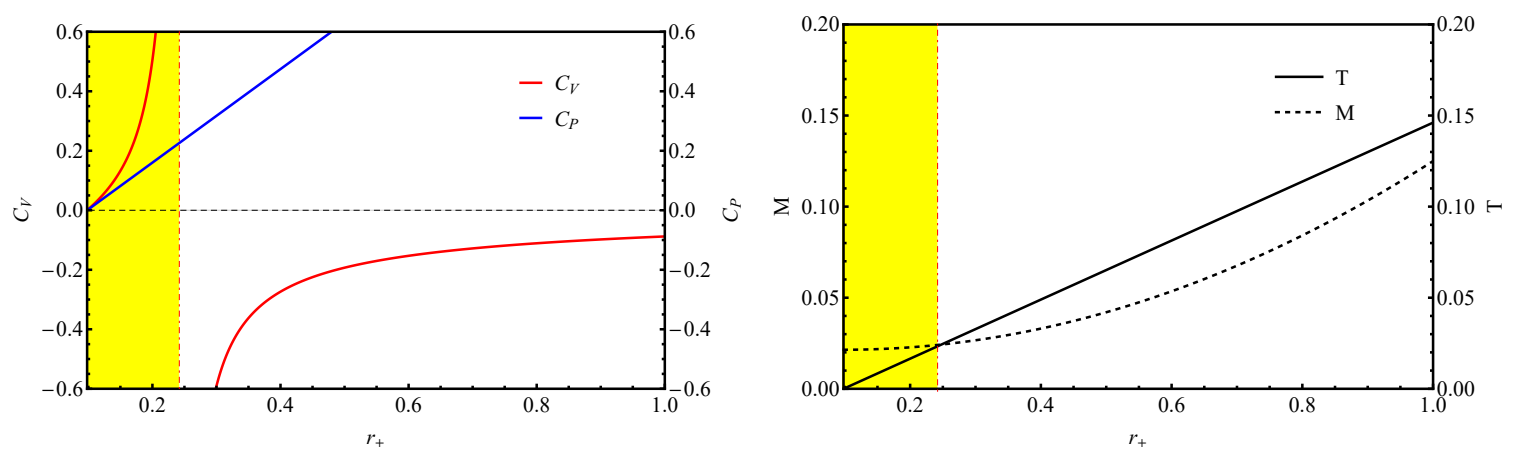

(d) $C_{V} \& C_{P}$ vs. $r_{+}$and $M \& T$ vs. $r_{+}$for $b=0.1$.

FIG. 1: Plots of the heat capacity at constant volume $C_{V}$, the heat capacity at constant pressure $C_{P}$, the black hole mass $M$ and the black hole temperature $T$ against the black hole horizon radius $r_{+}$for 3D EBI AdS black holes with $Q=1=l$ and various values of $b$. The yellow regions denote the regions of interest, where $C_{V}$ and $C_{P}$ are both positive, and hence black holes can be free of thermodynamic instability. 

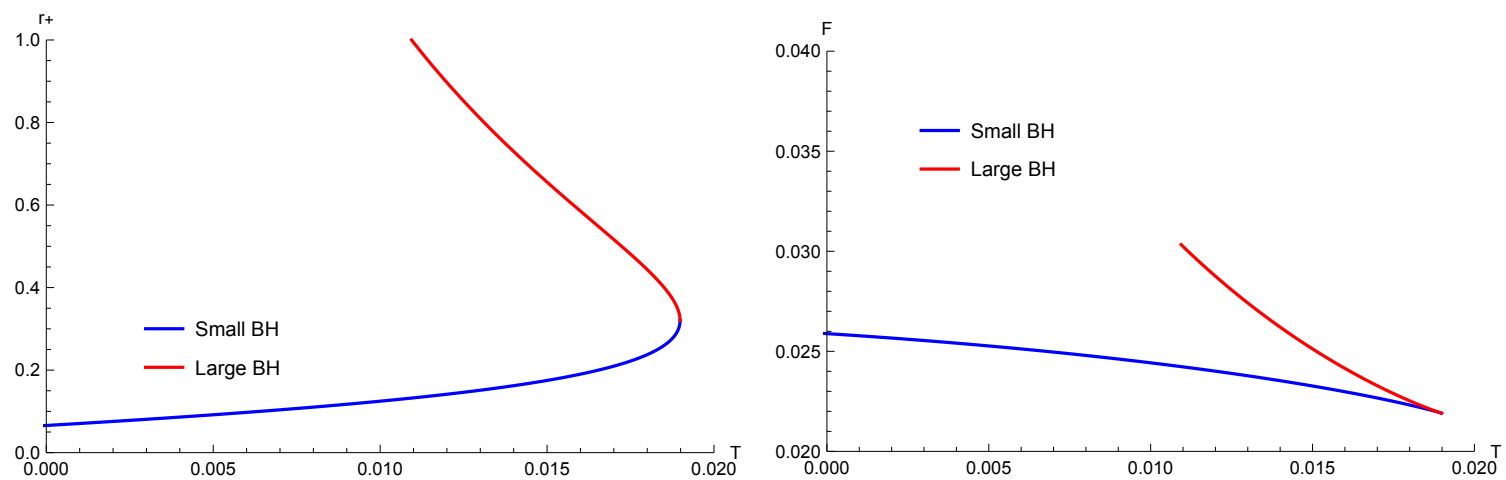

FIG. 2: Plots of the horizon radius $r_{+}$and the Helmholtz free energy $F$ against the black hole temperature $T$ for 3D EBI AdS black holes with fixed volume $V$. Here, we take $Q=1$ and $b=0.1$. The blue and red lines represent Small BH and Large BH, respectively. The specific heat at constant volume $C_{V}$ of Small/Large $\mathrm{BH}$ is positive/negative. As a result, $C_{V}$ is discontinuous at the maximum value of $T$.

black hole horizon radius $r_{+}$for 3D EBI AdS black holes with fixed pressure $l=1.0$, fixed charge $Q=1.0$ and various $b=0.1,0.5,1.69,100$. When $b=100$, non-linear electrodynamics effects are negligible, and hence behavior of 3D EBI AdS black holes closely resembles that of charged BTZ black holes. As shown in FIG. 1(a), $C_{P}$ is always positive whereas $C_{V}$ is always negative, which recovers the results of BTZ black holes [23]. As $b$ decreases to $b \simeq 1.69$, FIG. 1(b) exhibits that $C_{V}$ stays negative, and becomes large negative as $T$ goes to zero. Interestingly, for small enough values of $b$ (i.e., $b \lesssim 1.69$ ), our numerical results show that $C_{V}$ and $C_{P}$ can both be positive in some parameter region. In fact, when $b=0.5$ and 0.1, the regions where $C_{V}>0$ and $C_{P}>0$ are represented by yellow regions in FIGs. $1(\mathrm{c})$ and $1(\mathrm{~d})$, Note that the black hole temperature $T$ and mass $M$ are both positive in the yellow regions of FIGs. 1(c) and 1(d), which means that the 3D EBI AdS black hole solutions with $C_{V}>0$ and $C_{P}>0$ are physical. And FIGs. 1(c) \& 1(d) suggest that the conjecture violation region increases in size with decreasing parameter $b$. In short, we find that 3D EBI AdS black holes can violate the instability conjecture.

Interestingly, FIG. 1 shows that $C_{V}$ has a discontinuity for a small enough $b$. To investigate the nature of the discontinuity of $C_{V}$, we plot the horizon radius $r_{+}$and the Helmholtz free energy $F$ as functions of the black hole temperature $T$ with fixed volume $V$ in FIG. 2, where $Q=1$ and $b=0.1$. The left panel of FIG. 2 shows that, for a given $T$, there are two black hole solutions of different sizes, namely Large BH (red line) and Small BH (blue line). Moreover, the black hole temperature $T$ has a maximum $T_{\max }$, which corresponds to 
$\partial r_{+} /\left.\partial T\right|_{V}=0$. Note that $C_{V}$ can be rewritten as

$$
C_{V}=\left.\frac{\pi}{2} \frac{\partial r_{+}}{\partial T}\right|_{V},
$$

where we use Eq. (6) for the entropy $S$. Therefore, Large/Small BH has a negative/positive $C_{V}$, which goes to negative/positive infinity as $T$ approaches $T_{\max }$. In short, the discontinuity of $C_{V}$ corresponds to the maximum value of the black hole temperature, where the two black hole phases (i.e., Large BH and Small BH) merge. The right panel of FIG. 2 displays that the free energy of Small BH is always smaller than that of Large BH, which indicates that there is no phase transition. Our results suggest that, at a constant volume, 3D EBI AdS black holes with positive $C_{V}$ are globally stable.

\section{CONCLUSION AND DISCUSSION}

In this paper, considering 3D EBI AdS black holes, we tested the instability conjecture: super-entropic black holes always have $C_{V}<0$ or $C_{P}<0$ whenever $C_{V}>0$, making them unstable in extended gravitational thermodynamics. This conjecture was tested and found satisfied for a large class of super-entropic solutions [11, 23, 24]. After showing 3D EBI AdS black holes are super-entropic, we found that the black holes satisfy the instability conjecture when $b$ is large enough (i.e., non-linear electrodynamics effects are inessential). However, when non-linear electrodynamics effects play an important role, our numerical results (see FIG. 1) showed that there exists some parameter region, where both $C_{V}>0$ and $C_{P}>0$, and hence provided a counter example to the instability conjecture. In addition, it was suggested that the violation region will increase as non-linear electrodynamics effects become stronger.

It is worthwhile pointing out that for $d \geq 4$ dimension, the thermodynamic volume of EBI AdS black holes is just the naive geometric volume $V=(d-1)^{-1} \omega_{d-2} r_{+}^{d-1}$, which means that $R=1$, and hence higher dimensional EBI AdS black holes are not super-entropic. On the other hand, for higher dimensional EBI AdS black holes, the entropy $S$ and the volume $V$ are not independent, which leads to the constant volume specific heat $C_{V}=0[62]$. 


\section{Acknowledgments}

We are grateful to Wei Hong and Yucheng Huang for useful discussions and numerical analysis. This work is supported in part by NSFC (Grant No. 11005016, 11875196 and 11375121).

\section{Appendix A: Derivation of Eq. (17)}

In this appendix, we present a derivation of Eq. (17), which starts from the definition of the adiabatic compressibility and the isothermal bulk modulus. In fact, the adiabatic compressibility $\beta_{S}$ and the isothermal bulk modulus $\kappa_{T}$ are defined by

$$
\beta_{S} \equiv-\left.\frac{1}{V} \frac{\partial V}{\partial P}\right|_{S} \text { and } \kappa_{T} \equiv-\left.V \frac{\partial P}{\partial V}\right|_{T}
$$

respectively. Using properties of partial derivatives, one can rewrite $\beta_{S}$ as

$$
\beta_{S}=-\left.\left.\frac{1}{V} \frac{\partial V}{\partial S}\right|_{P} \frac{\partial S}{\partial P}\right|_{V}=-\frac{1}{V} \frac{\left.\frac{\partial S}{\partial P}\right|_{V}}{\left.\frac{\partial S}{\partial V}\right|_{P}}=-\frac{1}{V} \frac{\left.\left.\frac{\partial S}{\partial T}\right|_{V} \frac{\partial T}{\partial P}\right|_{V}}{\left.\left.\frac{\partial S}{\partial T}\right|_{P} \frac{\partial T}{\partial V}\right|_{P}}
$$

On the other hand, the specific heat at constant pressure $C_{P}$ and the specific heat at constant volume $C_{V}$ are defined by

$$
\left.C_{P} \equiv T \frac{\partial S}{\partial T}\right|_{P} \text { and }\left.C_{V} \equiv T \frac{\partial S}{\partial T}\right|_{V}
$$

respectively. Consequently, Eq. (A2) reduces to

$$
\beta_{S}=\frac{C_{V}}{C_{P}}\left(-\left.\left.\frac{1}{V} \frac{\partial T}{\partial P}\right|_{V} \frac{\partial V}{\partial T}\right|_{P}\right)=\frac{C_{V}}{C_{P}}\left(-\left.\frac{1}{V} \frac{\partial V}{\partial P}\right|_{T}\right)=\frac{C_{V}}{C_{P}} \frac{1}{\kappa_{T}},
$$

which leads to

$$
\frac{C_{P}}{C_{V}}=\frac{1}{\beta_{S} \kappa_{T}}
$$

[1] J. D. Bekenstein, Black holes and entropy, Phys. Rev. D 7 (1973), 2333-2346.

[2] J. D. Bekenstein, Generalized second law of thermodynamics in black hole physics, Phys. Rev. D 9 (1974), 3292-3300.

[3] S. W. Hawking, Particle Creation by Black Holes, Commun. Math. Phys. 43 (1975), 199-220.

[4] S. W. Hawking, Black Holes and Thermodynamics, Phys. Rev. D 13 (1976), 191-197. 
[5] D. Grumiller, R. McNees and J. Salzer, Black holes and thermodynamics - The first half century, Fundam. Theor. Phys. 178 (2015), 27-70.

[6] D. Kastor, S. Ray and J. Traschen, Enthalpy and the Mechanics of AdS Black Holes, Class. Quant. Grav. 26 (2009), 195011.

[7] S. Wang, S. Q. Wu, F. Xie and L. Dan, The First laws of thermodynamics of the $(2+1)$ dimensional BTZ black holes and Kerr-de Sitter spacetimes, Chin. Phys. Lett. 23 (2006), 1096-1098.

[8] Y. Sekiwa, Thermodynamics of de Sitter black holes: Thermal cosmological constant, Phys. Rev. D 73 (2006), 084009.

[9] M. Cvetic, G. W. Gibbons, D. Kubiznak and C. N. Pope, Black Hole Enthalpy and an Entropy Inequality for the Thermodynamic Volume, Phys. Rev. D 84 (2011), 024037.

[10] B. P. Dolan, D. Kastor, D. Kubiznak, R. B. Mann and J. Traschen, Thermodynamic Volumes and Isoperimetric Inequalities for de Sitter Black Holes, Phys. Rev. D 87 (2013) no.10, 104017.

[11] R. A. Hennigar, D. Kubizuk and R. B. Mann, Entropy Inequality Violations from Ultraspinning Black Holes, Phys. Rev. Lett. 115 (2015) no.3, 031101.

[12] D. Klemm, Four-dimensional black holes with unusual horizons, Phys. Rev. D 89 (2014) no.8, 084007.

[13] S. M. Noorbakhsh and M. H. Vahidinia, Extremal Vanishing Horizon Kerr-AdS Black Holes at Ultraspinning Limit, JHEP 01 (2018), 042.

[14] R. A. Hennigar, D. Kubizuk, R. B. Mann and N. Musoke, Ultraspinning limits and superentropic black holes, JHEP 06 (2015), 096.

[15] S. M. Noorbakhsh and M. Ghominejad, Higher Dimensional Charged AdS Black Holes at Ultra-spinning Limit and Their 2d CFT Duals, arXiv:1702.03448 [hep-th].

[16] M. Appels, L. Cuspinera, R. Gregory, P. Krtouš and D. Kubizňák, Are "Superentropic" black holes superentropic?, JHEP 02 (2020), 195.

[17] C. V. Johnson, V. L. Martin and A. Svesko, Microscopic description of thermodynamic volume in extended black hole thermodynamics, Phys. Rev. D 101 (2020) no.8, 086006.

[18] M. T. N. Imseis, A. A. Balushi and R. B. Mann, Null Hypersurfaces in Kerr-Newman-AdS Black Hole and Super-Entropic Black Hole Spacetimes, arXiv:2007.04354 [gr-qc].

[19] M. Sinamuli and R. B. Mann, Super-Entropic Black Holes and the Kerr-CFT Correspondence, JHEP 08 (2016), 148. 
[20] D. Wu, P. Wu, H. Yu and S. Q. Wu, Notes on the thermodynamics of superentropic AdS black holes, Phys. Rev. D 101 (2020) no.2, 024057.

[21] D. Wu, P. Wu, H. Yu and S. Q. Wu, Are ultraspinning Kerr-Sen- AdS 4 black holes always superentropic?, Phys. Rev. D 102 (2020) no.4, 044007.

[22] A. M. Frassino, R. B. Mann and J. R. Mureika, Lower-Dimensional Black Hole Chemistry, Phys. Rev. D 92 (2015) no.12, 124069.

[23] C. V. Johnson, Instability of Super-Entropic Black Holes in Extended Thermodynamics, Mod. Phys. Lett. A 33 (2020), 2050098.

[24] W. Cong and R. B. Mann, Thermodynamic Instabilities of Generalized Exotic BTZ Black Holes, JHEP 11 (2019), 004.

[25] X. H. Feng, H. S. Liu, W. T. Lu and H. Lu, Horndeski Gravity and the Violation of Reverse Isoperimetric Inequality, Eur. Phys. J. C 77 (2017) no.11, 790.

[26] S. Noda and Y. C. Ong, Null Hypersurface Caustics, Closed Null Curves, and Super-Entropy, arXiv:2009.13448 [gr-qc]].

[27] S. Boudet, F. Bombacigno, G. Montani and M. Rinaldi, Super-entropic black hole with Immirzi hair, arXiv:2012.02700 [gr-qc]].

[28] Z. M. Xu, The correspondence between thermodynamic curvature and isoperimetric theorem from ultraspinning black hole, Phys. Lett. B 807 (2020), 135529.

[29] D. Wu, S. Q. Wu, P. Wu and H. Yu, Entropy inequality is not always violated by the ultraspinning dyonic Kerr-Sen-AdS4 black holes, arXiv:2010.13518 [gr-qc].

[30] M. Banados, C. Teitelboim and J. Zanelli, The Black hole in three-dimensional space-time, Phys. Rev. Lett. 69 (1992), 1849-1851.

[31] M. Banados, M. Henneaux, C. Teitelboim and J. Zanelli, Geometry of the $(2+1)$ black hole, Phys. Rev. D. Phys. Rev. D 48 (1993), 1506-1525 [erratum: Phys. Rev. D 88 (2013), 069902].

[32] C. V. Johnson, Specific Heats and Schottky Peaks for Black Holes in Extended Thermodynamics, Class. Quant. Grav. 37 (2020) no.5, 054003.

[33] M. Born and L. Infeld, Foundations of the new field theory, Proc. Roy. Soc. Lond. A 144 (1934) no.852, 425-451.

[34] G. W. Gibbons, Aspects of Born-Infeld theory and string / M theory, AIP Conf. Proc. 589 (2001) no.1, 324-350.

[35] E. S. Fradkin and A. A. Tseytlin, Nonlinear Electrodynamics from Quantized Strings, Phys. 
Lett. B 163 (1985), 123-130.

[36] A. A. Tseytlin, Vector Field Effective Action in the Open Superstring Theory, Nucl. Phys. B 276 (1986), 391.

[37] R. R. Metsaev, M. Rakhmanov and A. A. Tseytlin, The \{Born-Infeld $\}$ Action as the Effective Action in the Open Superstring Theory, Phys. Lett. B 193 (1987), 207-212.

[38] T. K. Dey, Born-Infeld black holes in the presence of a cosmological constant, Phys. Lett. B 595 (2004), 484-490.

[39] R. G. Cai, D. W. Pang and A. Wang, Born-Infeld black holes in (A)dS spaces, Phys. Rev. D 70 (2004), 124034.

[40] Y. S. Myung, Y. W. Kim and Y. J. Park, Thermodynamics of Einstein-Born-Infeld black holes in three dimensions, Phys. Rev. D 78 (2008), 044020.

[41] M. Cataldo and A. Garcia, Three dimensional black hole coupled to the Born-Infeld electrodynamics, Phys. Lett. B 456 (1999), 28-33.

[42] S. Fernando and D. Krug, Charged black hole solutions in Einstein-Born-Infeld gravity with a cosmological constant, Gen. Rel. Grav. 35 (2003), 129-137.

[43] S. H. Hendi, B. Eslam Panah and S. Panahiyan, Einstein-Born-Infeld-Massive Gravity: adSBlack Hole Solutions and their Thermodynamical properties, JHEP 11 (2015), 157.

[44] R. Banerjee, S. Ghosh and D. Roychowdhury, New type of phase transition in Reissner Nordström-AdS black hole and its thermodynamic geometry, Phys. Lett. B 696 (2011), 156-162.

[45] S. Li, H. Lu and H. Wei, Dyonic (A)dS Black Holes in Einstein-Born-Infeld Theory in Diverse Dimensions, JHEP 07 (2016), 004.

[46] A. Dehyadegari and A. Sheykhi, Reentrant phase transition of Born-Infeld-AdS black holes, Phys. Rev. D 98 (2018) no.2, 024011.

[47] M. Aiello, R. Ferraro and G. Giribet, Exact solutions of Lovelock-Born-Infeld black holes, Phys. Rev. D 70 (2004), 104014.

[48] S. Fernando, Thermodynamics of Born-Infeld-anti-de Sitter black holes in the grand canonical ensemble, Phys. Rev. D 74 (2006), 104032.

[49] S. Gunasekaran, R. B. Mann and D. Kubiznak, Extended phase space thermodynamics for charged and rotating black holes and Born-Infeld vacuum polarization, JHEP 11 (2012), 110.

[50] D. C. Zou, S. J. Zhang and B. Wang, Critical behavior of Born-Infeld AdS black holes in the extended phase space thermodynamics, Phys. Rev. D 89 (2014) no.4, 044002. 
[51] X. X. Zeng, X. M. Liu and L. F. Li, Phase structure of the Born-Infeld-anti-de Sitter black holes probed by non-local observables, Eur. Phys. J. C 76 (2016) no.11, 616.

[52] P. Wang, H. Wu and H. Yang, Thermodynamics and Phase Transitions of Nonlinear Electrodynamics Black Holes in an Extended Phase Space, JCAP 04 (2019), 052.

[53] P. Wang, H. Wu and H. Yang, Thermodynamics and Phase Transition of a Nonlinear Electrodynamics Black Hole in a Cavity, JHEP 07 (2019), 002.

[54] R. Banerjee, S. Gangopadhyay, D. Roychowdhury and A. Lala, Holographic s-wave condensate with nonlinear electrodynamics: A nontrivial boundary value problem, Phys. Rev. D 87 (2013) no.10, 104001.

[55] J. Tao, P. Wang and H. Yang, Testing holographic conjectures of complexity with Born-Infeld black holes, Eur. Phys. J. C 77 (2017) no.12, 817.

[56] S. Bi, M. Du, J. Tao and F. Yao, Joule-Thomson Expansion of Born-Infeld AdS Black Holes, arXiv:2006.08920 [gr-qc]].

[57] H. Ma and J. Li, Dirac quasinormal modes of Born-Infeld black hole spacetimes, Chin. Phys. C 44 (2020) no.9, 095102.

[58] E. Caceres, P. H. Nguyen and J. F. Pedraza, Holographic entanglement entropy and the extended phase structure of STU black holes, JHEP 09 (2015), 184extbf48 (1993), 1506-1525.

[59] C. V. Johnson, Thermodynamic Volumes for AdS-Taub-NUT and AdS-Taub-Bolt, Class. Quant. Grav. 31 (2014) no.23, 235003.

[60] W. G. Brenna, R. B. Mann and M. Park, Mass and Thermodynamic Volume in Lifshitz Spacetimes, Phys. Rev. D 92 (2015) no.4, 044015.

[61] S. M. Noorbakhsh and M. Ghominejad, Ultra-Spinning Gauged Supergravity Black Holes and their Kerr/CFT Correspondence, Phys. Rev. D 95 (2017) no.4, 046002.

[62] B. P. Dolan, The cosmological constant and the black hole equation of state, Class. Quant. Grav. 28 (2011), 125020. 